OPEN ACCESS

Edited by:

Gang Zhang,

Institute of High Performance Computing (A*STAR), Singapore

Reviewed by:

Chengyong Zhong

Chongqing University of Posts and

Telecommunications, China

Weifeng $\mathrm{Li}$,

Shandong University, China

*Correspondence:

Guochun Yang

yanggc468@nenu.edu.cn orcid.org/0000-0003-3083-472X

Aitor Bergara

a.bergara@ehu.eus

Specialty section:

This article was submitted to

Condensed Matter Physics,

a section of the journal

Frontiers in Physics

Received: 20 June 2018

Accepted: 23 August 2018 Published: 19 September 2018

Citation:

Zheng S, Zhang S, Sun Y, Zhang J,

Lin J, Yang G and Bergara A (2018)

Structural and Superconducting

Properties of Tungsten Hydrides

Under High Pressure.

Front. Phys. 6:101.

doi: 10.3389/fphy.2018.00101

\section{Structural and Superconducting Properties of Tungsten Hydrides Under High Pressure}

\author{
Shuang Zheng ${ }^{1}$, Shoutao Zhang ${ }^{1}$, Yu Sun ${ }^{1}$, Jing Zhang ${ }^{1}$, Jianyan Lin ${ }^{1}$, Guochun Yang ${ }^{1 *}$ \\ and Aitor Bergara ${ }^{2,3,4 *}$
}

${ }^{1}$ Centre for Advanced Optoelectronic Functional Materials Research and Key Laboratory for UV Light-Emitting Materials and Technology of Ministry of Education, Northeast Normal University, Changchun, China, ${ }^{2}$ Departmento de Física de la Materia Condensada, Universidad del País Vasco, UPV/EHU, Bilbao, Spain, ${ }^{3}$ Donostia International Physics Center, Donostia, Spain, ${ }^{4}$ Centro de Física de Materiales, Centro Mixto CSIC-UPV/EHU, Donostia, Spain

Unveiling the relation between crystal structure and superconductivity of hydrides becomes a fascinating research area in chemistry and condensed-mater physics. Although much efforts have been made to study chemical reaction between tungsten and hydrogen, the crystal structures, superconductivity, and phase diagram of tungsten hydrides under high pressure have not been fully explored and built thus far. In this work, we carried out extensive structural search on $\mathrm{W}-\mathrm{H}$ binary compounds through first-principles swarm-intelligence structural search calculations. Besides reproducing the known $\mathrm{W}-\mathrm{H}$ compounds, a new stoichiometry $\mathrm{WH}_{5}$ with $\mathrm{P} 6 \mathrm{~mm}$ symmetry becomes stable above $230.2 \mathrm{GPa}$. Intriguingly, $P 6 \mathrm{~mm} \mathrm{WH}_{5}$ shows a critical temperature $\left(T_{\mathrm{c}}\right)$ value of $60.8 \mathrm{~K}$, which is much higher than $31.6 \mathrm{~K}$ in $\mathrm{WH}_{6}$. This finding is different from the knowledge of compounds with higher $\mathrm{H}$ content exhibiting higher $T_{\mathrm{C}}$, which might be attributed to the appearance of unique $\mathrm{H}$ network and tetrahedron $\mathrm{H}$ units in $\mathrm{WH}_{5}$. Electronic property and superconductivity of the other tungsten hydrides are also investigated. The built pressure-composition phase diagram provides some useful information for experimental synthesis.

Keywords: tungsten hydride, superconductivity, high pressure, structure prediction, density functional theory

\section{INTRODUCTION}

An important objective in condensed-matter physics and chemistry is to prepare hydrogen-rich (H-rich) compounds because of their broad applications and scientific significance [1-5]. In more detail, $\mathrm{H}$-rich compounds are the excellent hydrogen energy storage materials [6-8] and hightemperature superconductors [9-11]. On the other hand, H-rich compounds, especially at high pressures, contain various kinds of chemical bonds (e.g., covalent, ionic, and van der Waals bonds) [12] and diverse hydrogen structure units [e.g., unpaired $\mathrm{H}$ [13], $\mathrm{H}_{2}$ [14], $\mathrm{H}_{3}$ units [15], and 3D hydrogen network resembling metallic atomic hydrogen [16, 17]], providing opportunity for us to fully understand physical and chemical properties of hydrogen.

Wigner et al. predicted that metalized solid hydrogen is the potential room-temperature superconductor due to its high phonon frequencies and strong electron-phonon coupling [18]. However, hydrogen metallization is rather difficult such as extreme high pressure [19, 20]. Fortunately, Ashcroft proposed that metallization of hydrogen-rich compounds needs relatively 
lower pressures [10], which also show high-temperature superconductivity $[1,2,13,21,22]$. Among the various $\mathrm{H}$-rich compounds, metal hydrides as the potential superconductors have drawn much attentions because of large density of states at the Fermi level, high phonon frequencies, and strong electronphonon coupling $[23,24]$. Great progress has been made in metal hydrides [11, 25-28]. Interestingly, some of metal hydrides under high pressure show large $T_{\mathrm{c}}$ values $[2,29,30]$. For example, the $T_{\mathrm{c}}$ for $\mathrm{LiH}_{6}$ reaches $82 \mathrm{~K}$ at $300 \mathrm{GPa}$ [31]. The C2/c phase of $\mathrm{KH}_{6}$ is a superconductor with an estimated $T_{\mathrm{c}}$ of $58.66-69.84 \mathrm{~K}$ at 166 $\mathrm{GPa}$ [5]. Recent theoretical calculation revealed that the potential high-temperature superconductor of $\mathrm{CaH}_{6}$ has a $T_{\mathrm{c}}$ of $220-235 \mathrm{~K}$ at $150 \mathrm{GPa}$ [2]. For $\mathrm{Mg}-\mathrm{H}$ system, $\mathrm{MgH}_{6}$ shows a $T_{\mathrm{c}}$ value of $263 \mathrm{~K}$ at $300 \mathrm{GPa}$ [29]. Moreover, a theoretical work indicated that $T_{\mathrm{c}}$ value of $34.1-40.5 \mathrm{~K}$ for $R-3 \mathrm{InH}_{3}$ at $200 \mathrm{GPa}$ [15]. The $P 6 / \mathrm{mmm} \mathrm{SnH}_{4}$ was predicted to be a superconductor with a high $T_{\mathrm{c}}(73 \mathrm{~K}$ at $120 \mathrm{GPa})[21]$.

Among the various hydrides, transition metal hydrides have drawn a growing interest due to their lower synthesis pressure with respect to the other hydrides, multiple stoichiometries, catalytic transformations, and superconductivity [32-34]. These unique properties can be attributed to the diverse $d$ electron configurations of transition metals. More interestingly, transition metal hydrides with higher hydrogen content become stabilized with increasing pressure $[35,36]$, consisting of diverse hydrogen structure units and exhibiting intriguing properties [6, 11-16]. For instance, $\mathrm{FeH}_{x}(x=1-4)$ compounds have been reported [37-40]. Very recently, Pepin et al. reported synthesis of $\mathrm{FeH}_{5}$ containing non-bonded hydrogen atoms above $130 \mathrm{GPa}$, which opens up a strategy to access metallic atomic hydrogen [41]. It is worth noting that finding metallic atomic hydrogen at a low pressure is the long-pursuit targets. In addition, $\mathrm{FeH}_{5}$ shows a superconducting transition temperature of $51 \mathrm{~K}$ [42]. Inspired by these findings, Kvashnin et al. [43] and Zhang et al. [44] independently studied phase diagram and electronic properties of $\mathrm{Fe}-\mathrm{H}$ binary compounds with diverse stoichiometries at high pressures. A new H-rich compound, $\mathrm{FeH}_{6}$, was identified. Notably, $\mathrm{Cmmm} \mathrm{FeH}_{6}$ is metallic [43], whereas $\mathrm{C}_{2} / \mathrm{c} \mathrm{FeH}$ is nonmetallic [44], which becomes rare example of pressure-induced non-metallicity among the transition-metal hydrides [45]. On the other hand, parts of transition metal hydrides especially at high pressures are metallization and exhibit superconductivity $[9,11,13,22,33,35]$. In more detail, Li et al. revealed that the $\mathrm{YH}_{4}$ and $\mathrm{YH}_{6}$ are potential superconductors with the estimated $T_{\mathrm{c}}$ values of $84-95 \mathrm{~K}$ and $251-264 \mathrm{~K}$ at $120 \mathrm{GPa}$ [13]. It is found that $\mathrm{TaH}_{6}$ with an estimated $T_{\mathrm{c}}$ of $124.2-135.8 \mathrm{~K}$ under pressure is a candidate as a high-temperature superconductor [35]. As for vanadium hydrides, the high-pressure structures of $\mathrm{VH}_{5}$ and $\mathrm{VH}_{8}$ have been explored, their $T_{\mathrm{c}}$ values were predicted to be 18.5 and $71.4 \mathrm{~K}$ at $200 \mathrm{GPa}$ due to the strong electron-phonon coupling (EPC) [36]. Consequently, these studies motivated us to search high-temperature superconductors of transition metal hydrides through compression.

Tungsten (W) is one of transition metal elements. At atmospheric pressure, molecular tungsten hydrides such as $\mathrm{WH}_{2}$, $\mathrm{WH}_{4}$, and molecular complex $\mathrm{WH}_{4}\left(\mathrm{H}_{2}\right)_{4}$ were identified using spectroscopy at low temperatures and in neon matrix [46, 47].
However, they are unstable with respect to hydrogen and bulk $\mathrm{W}$ at ambient conditions. In other words, there is no stable solid tungsten hydride at ambient conditions. Once, it was thought that $\mathrm{W}$ hardly reacts with $\mathrm{H}_{2}$ under pressure [48], and was used as a sealing material in high-pressure experiment [19]. However, finding near-stoichiometric WH above $~ 30$ $\mathrm{GPa}$ arouses much attention to studying tungsten hydrides at high pressures [49]. Specifically, variable composition structural prediction was performed to investigate structures and phase stability of $\mathrm{WH}_{n}(n=1-6,8,10$, and 12$)$ up to $150 \mathrm{GPa}[46,50]$. Three $\mathrm{W}-\mathrm{H}$ compounds (i.e., $\mathrm{WH}, \mathrm{WH}_{2}$, and $\mathrm{WH}_{6}$ ) are found to be stable [46, 51]. Subsequently, Gregoryanz et al. studied the formation, decomposition, and mechanical properties of tungsten hydrides in a diamond anvil cell up to $115 \mathrm{GPa}$ [52]. Notably, these studies mainly focus on structures and stabilities of tungsten hydrides below $150 \mathrm{GPa}$. To the best of our knowledge, their electronic properties and superconductivity have not been explored so far.

Generally speaking, most of metal hydrides exhibit superconductivity, and high pressure is in favor of stabilizing H-rich compounds [e.g., $\mathrm{LaH}_{8}$ [11], $\mathrm{BaH}_{6}$ [53], $\mathrm{YH}_{10}$ [16], and $\mathrm{LaH}_{10}$ [17]]. On the other hand, H-rich compounds show larger $T_{\mathrm{c}}$ value [54]. Therefore, we expect that tungsten hydrides with a higher hydrogen content become stable under high pressure. Moreover, understanding electronic structures and superconductivity of tungsten hydrides is important to explore their potential applications. In this work, we studied the structural stabilities of tungsten hydrides containing various $\mathrm{W}_{x} \mathrm{H}_{y}(x=1, y=1-12 ; x=2, y=1)$ compositions at pressures of 100,200, and $300 \mathrm{GPa}$ via first-principle structure search calculations. Besides reproducing the reported tungsten hydrides, we find a new stable stoichiometry $\mathrm{WH}_{5}$ consisting of unique $\mathrm{H}$ network and tetrahedron $\mathrm{H}$ units, exhibiting the highest $T_{\mathrm{c}}$ value among the tungsten hydrides.

\section{COMPUTATIONAL DETAILS}

The structural prediction of $\mathrm{W}-\mathrm{H}$ system was performed via particle swarm optimization as implemented in CALYPSO code $[55,56]$ at the selected pressures of 100,200 , and $300 \mathrm{GPa}$. The main merit of this code is capable of finding the ground-state structures only depending on the given chemical composition. Its efficiency and validity have been proven by applying different kinds of systems, ranging from elemental solids to binary and ternary compounds [2, 57-62]. Considering these advantages, CALYPSO becomes a leading structure prediction method in the field. Then, the structural relaxations for the considered $\mathrm{W}$ $\mathrm{H}$ compounds were performed through the Broyden-FletcherGoldfarb-Shanno (BFGS) [63] method as implemented in Cambridge Serial Total Energy Package (CASTEP) program [64]. The Perdew-Burke-Ernzerh (PBE) of generalized gradient approximation (GGA) was selected for the exchange-correlation functional [65]. The electron-ion interaction was described by using the ultrasoft pseudopotential [66] plane-wave method, in which $5 s^{2} 5 p^{6} 5 d^{4} 6 s^{2}$ and $1 s^{1}$ were taken as valence electrons for $\mathrm{W}$ and $\mathrm{H}$ atoms, respectively. The kinetic cutoff energy 
of $400 \mathrm{eV}$ and the Monkhorst-Pack scheme [67] with a $k$ point grid of $2 \pi \times 0.03 \AA^{-1}$ were chosen to ensure that the total energy well-converged. The phonon spectra and the electron-phonon coupling were calculated within the framework of the linear-response theory as carried out in the QuantumESPRESSO package [68]. The kinetic energy cutoff for wavefunction expansion is chosen as $80 \mathrm{Ry}$. A $k$-point mesh of $15 \times$ $15 \times 14$ and a $q$-point mesh of $5 \times 5 \times 2$ for $\mathrm{WH}_{5}$ were used in the first Brillouin zone. More detailed calculated information can be found in the Supporting Information.

\section{RESULTS AND DISCUSSION}

To obtain higher $T_{\mathrm{c}}$ value, high hydrogen content in compounds is one of the necessary prerequisites $[9,11,54]$. As a consequence, we carried out the crystal structure search on $\mathrm{W}_{x} \mathrm{H}_{y}(x=1$, $y=1-12 ; x=2, y=1)$ stoichiometries at the selected pressures of 100, 200, and $300 \mathrm{GPa}$ through first-principles swarm structure searching calculations. First, based on the predicted stable structures, we studied the relative phase stability of each stoichiometric compound by calculating its enthalpy of formation $\left(\Delta H=\left[H\left(\mathrm{~W}_{x} \mathrm{H}_{y}\right)-x H(\mathrm{~W})-y H\left(\mathrm{H}_{2}\right) / 2\right] /(x\right.$ $+y)$ ) relative to elemental $\mathrm{W}$ and $\mathrm{H}_{2}$ solids at the selected pressures. Here, $H\left(\mathrm{~W}_{x} \mathrm{H}_{y}\right)$ is the enthalpy of considered structure, $H(\mathrm{~W})$ and $H\left(\mathrm{H}_{2}\right)$ are the enthalpies of elemental W and $\mathrm{H}_{2}$ solids, respectively. Then, convex hull data for considered $\mathrm{W}-\mathrm{H}$ compositions at selected pressures are constructed using enthalpy of formation per atom $\Delta H$ for each composition to determine its stability (Figure 1A). In general, a structure whose enthalpy of formation sits on the convex hull (i.e., solid line) is thermodynamically and is experimentally synthesizable. At $100 \mathrm{GPa}$, the known $\mathrm{WH}, \mathrm{WH}_{2}$, and $\mathrm{WH}_{4}$ are well-reproduced by our structural search, validating that our adopted method is suitable to the $\mathrm{W}-\mathrm{H}$ system. At $200 \mathrm{GPa}, \mathrm{WH}, \mathrm{WH}_{2}$, and $\mathrm{WH}_{6}$ are stable, whereas $\mathrm{WH}_{4}$ becomes unstable with respect to $\mathrm{WH}_{2}$ and $\mathrm{WH}_{6}$. With further compression, a new stoichiometry $\mathrm{WH}_{5}$, is thermodynamically stable in addition to $\mathrm{WH}$ and $\mathrm{WH}_{2}$ at 300 $\mathrm{GPa}$, whereas $\mathrm{WH}_{6}$ decomposes into $\mathrm{WH}_{5}$ and $\mathrm{H}_{2}$. It is noted that $\mathrm{WH}$ is the most stable composition in the whole considered pressure range. To provide more information for experimental synthesis, we determine the pressure stability region of $\mathrm{W}-\mathrm{H}$ compounds, as displayed in Figure 1B. Intriguingly, these stable compounds do not undergo any structural transitions. This is in sharp contrast with other transition metal hydrides, such as $\mathrm{VH}_{3}$ [71] and $\mathrm{ScH}_{6}$ [9].

$\mathrm{WH}_{5}$ becomes stable above $230.2 \mathrm{GPa}$ and stabilizes into a hexagonal structure (space group $P 6 \mathrm{~mm}$, one formula unit per cell, see Figure 2). This structure contains two inequivalent H's occupying $2 \mathrm{~b}(0.3333,0.6667,0.6985)$ and $3 c(0.0000,0.5000$, $0.0825)$ sites and one equivalent W's sitting at $1 \mathrm{a}(0.0000,0.0000$, 0.4728 ) position. Each $\mathrm{W}$ atom forms a twelve-fold coordination with $\mathrm{H}$ atoms (Figure 2A), having two different $\mathrm{W}-\mathrm{H}$ distances of 1.70 and $1.71 \AA$. Mulliken charge analysis [72] shows W atom transfers 0.17 e to each $\mathrm{H}$ atom, indicating that $\mathrm{W}-\mathrm{H}$ bonding is ionic. There are two kinds of $\mathrm{H}$ coordination. The $\mathrm{H}$ atom at $2 \mathrm{~b}$ site is coordinated by three $\mathrm{W}$ atoms, while

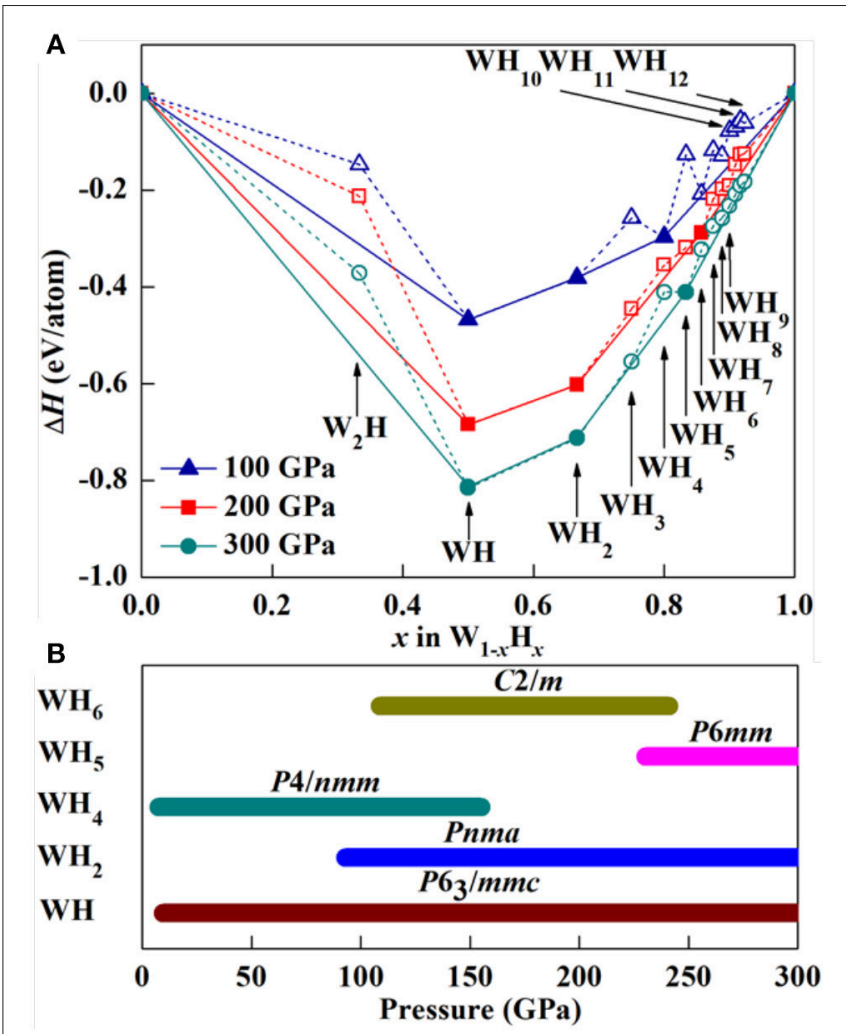

FIGURE 1 | (A) Phase stabilities of various W-H compounds at 100, 200, and $300 \mathrm{GPa}$. The elemental W solid phase with symmetry Im-3m [69], and $\mathrm{P6}_{3} / \mathrm{m}, \mathrm{C} 2 / \mathrm{c}, \mathrm{Cmca}-12$ elemental $\mathrm{H}_{2}$ solids [70] are adopted to calculate the formation of enthalpy. (B) Pressure-composition phase diagram for the $\mathrm{W}-\mathrm{H}$ compounds.

$\mathrm{H}$ atom at $3 \mathrm{c}$ site is shared by two $\mathrm{W}$ atoms (Figure $2 \mathbf{B}$ ). Notably, $\mathrm{H}$ atoms form intriguing $\mathrm{H}$ network with alternate triangle and hexagon rings along $c$ axis with equal $\mathrm{H}-\mathrm{H}$ distance of $1.38 \AA$ (Figure 2C), which is called a kagome lattice [7375]. Moreover, the $\mathrm{H}$ atom in the $a b$ plane constitutes vertexsharing tetrahedron units having $\mathrm{H}-\mathrm{H}$ distance of $1.28 \sim 1.38 \AA$ (Figure 2D). These $\mathrm{H}-\mathrm{H}$ distances in $\mathrm{WH}_{5}$ is much larger than $\mathrm{H}_{2}(0.74 \AA)[16]$ at ambient conditions and monatomic $\mathrm{H}(0.98$ $\AA$ ) at $500 \mathrm{GPa}$ [76], and comparable to lanthanum and yttrium hydrides $(1.02 \sim 1.54 \AA)$ [11]. indicating that $\mathrm{WH}_{5}$ might has high $T_{\mathrm{c}}$ value as observed in $\mathrm{CaH}_{6}$ [2], $\mathrm{LaH}_{8}$ [11], and $\mathrm{YH}_{10}$ [13].

Dynamic stability of a compound strongly correlates with its practical application. Thus, we calculated phonon spectra of $P 6 \mathrm{~mm}_{\mathrm{WH}} \mathrm{WH}_{5}$ as implemented in Quantum-ESPRESSO package (Figure 3A). The absence of any imaginary frequency in the entire Brillouin zone obviously confirms its dynamic stability. Based on the analysis of projected phonon density of states (PHDOS, Figure 3B), there are two separate regions. The high frequency modes (above $\sim 30 \mathrm{THz}$ ) mainly originate from the contribution of $\mathrm{H}$ atoms, whereas the low-frequency modes (below $\sim 15 \mathrm{THz}$ ) associate with $\mathrm{W}$ atoms, which is attributed to that $\mathrm{H}$ atom has a lighter atomic mass than W. Moreover, 


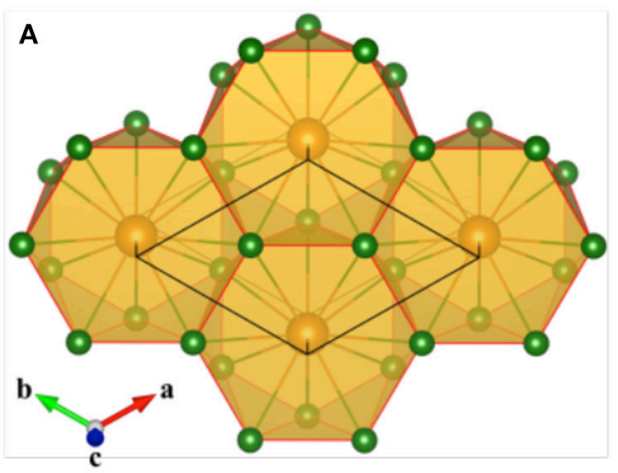

B

C

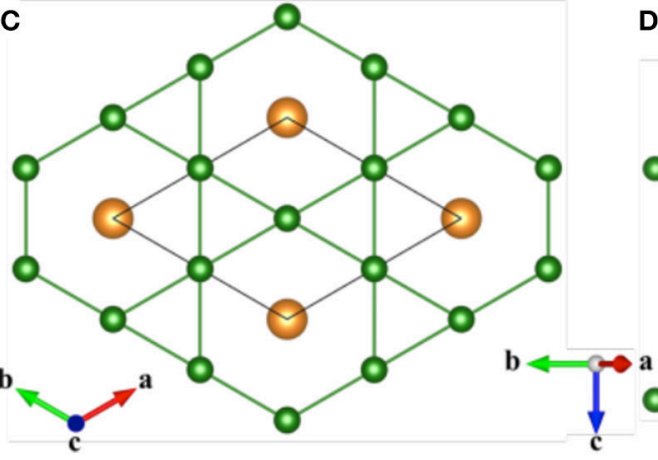

D
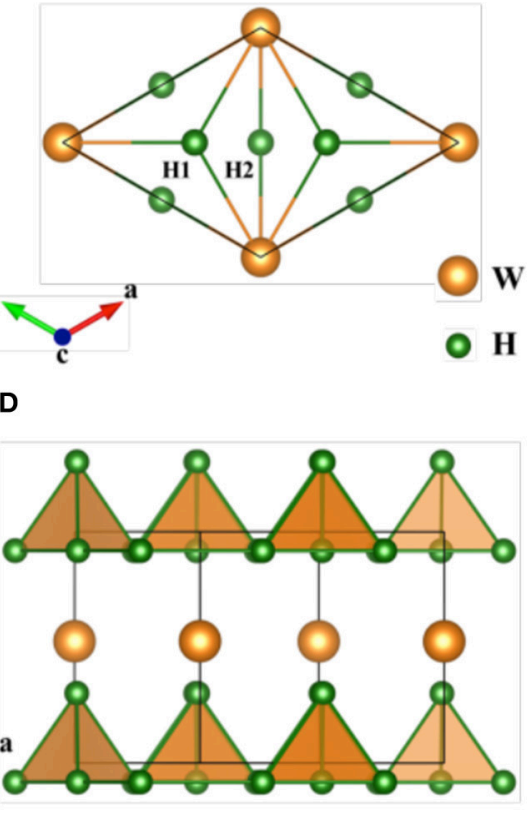

FIGURE 2 | Crystal structure of $\mathrm{WH}_{5}$ with $\mathrm{P} 6 \mathrm{~mm}$ symmetry at $300 \mathrm{GPa}$. (A) View of $\mathrm{W}-\mathrm{H}$ polyhedrons in $P 6 \mathrm{~mm}_{\mathrm{W}} \mathrm{WH}_{5}$. (B) Two inequivalent $\mathrm{H}$ atoms with three- and two-fold $\mathrm{W}$ atoms. (C) $\mathrm{H}$ network with alternate triangle and hexagon rings along $\mathrm{c}$ axis is a kagome lattice. (D) Vertex-sharing tetrahedron unit consisting of four $\mathrm{H}$ atoms in the ab plane.
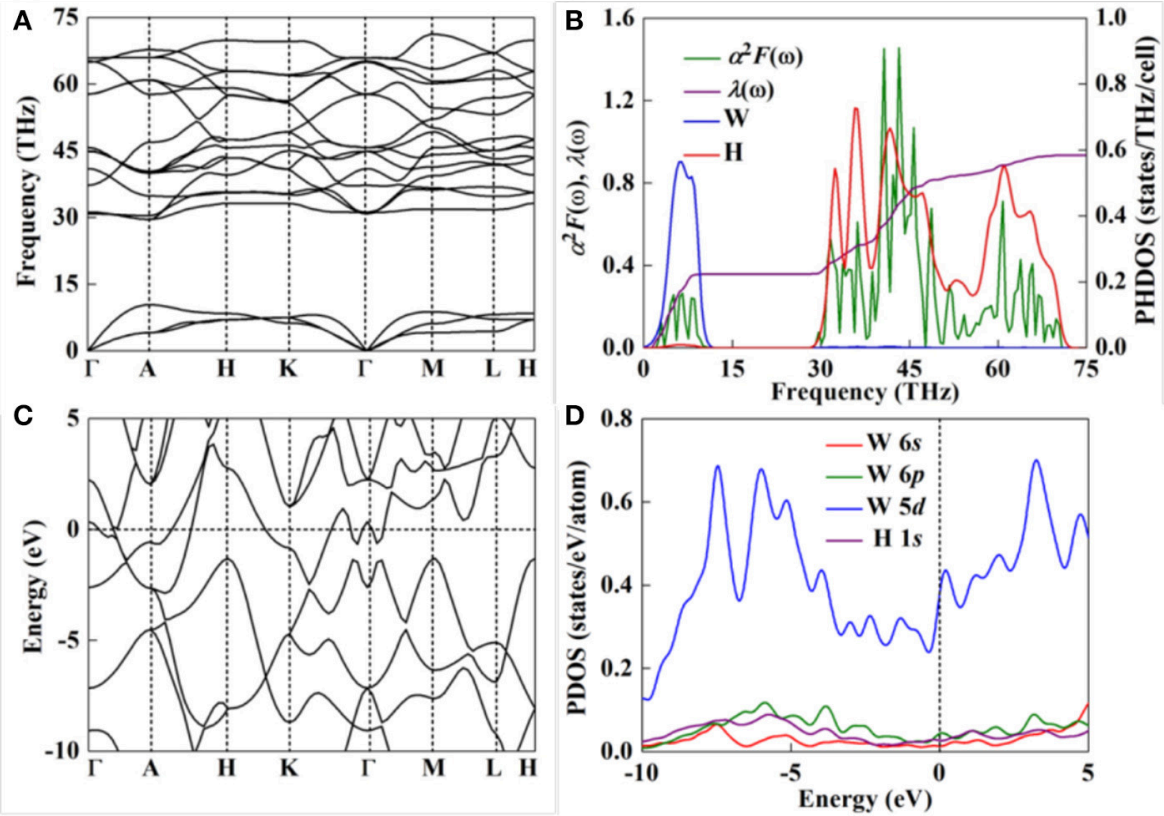

FIGURE 3 | (A) Phonon dispersion curve, (B) Eliashberg spectral function $\alpha^{2} F(\omega)$, electron-phonon coupling integration $\lambda(\omega)$, and projected phonon density of states (PHDOS) on W and $\mathrm{H}$ atoms, (C) electronic band structure, and (D) PDOS for $\mathrm{P} 6 \mathrm{~mm} \mathrm{WH}_{5}$ at $300 \mathrm{GPa}$. 
TABLE 1 | The logarithmic average phonon frequency $\omega_{\text {log }}$, the electronic density of states at the Fermi level $N\left(E_{f}\right)$, the electron-phonon coupling parameter $\lambda$, and the superconducting transition temperature $T_{\mathrm{C}}$ of $\mathrm{W}-\mathrm{H}$ compounds at selected pressures.

\begin{tabular}{|c|c|c|c|c|c|c|}
\hline Phases & $P(\mathrm{GPa})$ & $\lambda$ & $\omega_{\log }(\mathrm{K})$ & $\begin{array}{c}N\left(E_{f}\right) \\
\text { (states/Ry/cell) }\end{array}$ & $\begin{array}{c}T_{\mathrm{C}}(\mathrm{K}) \mu \\
\mu^{\star}=0.1\end{array}$ & $\begin{array}{c}T_{\mathrm{c}}(\mathrm{K}) \\
\mu^{*}=0.13\end{array}$ \\
\hline$P 6_{3} / m m c \mathrm{WH}$ & 300 & 0.454 & 517.356 & 7.502 & 4.175 & 2.247 \\
\hline $\mathrm{Pnma} \mathrm{WH}_{2}$ & 300 & 0.383 & 459.848 & 10.102 & 1.484 & 0.586 \\
\hline $\mathrm{P} 4 / \mathrm{nmm} \mathrm{WH}_{4}$ & 140 & 0.256 & 859.948 & 2.568 & 0.065 & 0.003 \\
\hline $\mathrm{P}_{6 m m} \mathrm{WH}_{5}$ & 300 & 0.929 & 982.292 & 3.610 & 60.792 & 51.302 \\
\hline $\mathrm{C} 2 / m \mathrm{WH}_{6}$ & 240 & 0.742 & 792.559 & 4.448 & 31.586 & 24.817 \\
\hline
\end{tabular}

$\mu^{*}$ is Coulomb pseudopotential.

$\mathrm{P6}_{3} / \mathrm{mmc} \mathrm{WH}, \mathrm{Pnma} \mathrm{WH}_{2}, P 4 / n m m \mathrm{WH}_{4}$, and $C 2 / m \mathrm{WH}_{6}$ are also dynamically stable (Figure S2).

Subsequently, we probe the electronic band structure and corresponding projected density of states (PDOS) of the stable $\mathrm{W}-\mathrm{H}$ compounds as depicted in Figures 3C,D and Figure S1. As we expected, all the $\mathrm{W}-\mathrm{H}$ compounds exhibit metallic character due to several bands across their Fermi levels. For these W$\mathrm{H}$ compounds, there is a common character of their electron properties (i.e., the main contribution to PDOS near the Fermi level comes from W $5 d$ orbital). Moreover, there is large overlap between $\mathrm{H} 1 s$ orbital and $\mathrm{W} 6 \mathrm{~s}$ or $6 p$ orbital. The appearance of "flat band-steep band" [3, 77, 78] at Fermi level and large $N\left(E_{\mathrm{f}}\right)$ indicate that $\mathrm{W}-\mathrm{H}$ compounds are the potential hightemperature superconductors [79].

Subsequently, we investigate their superconductivity based on Bardeen-Cooper-Schrieffer (BCS) theory [80]. Among the stable tungsten hydrides, $\mathrm{WH}_{5}$ has the largest electron-phonon coupling parameter, $\lambda$, which is comparable to those predicted for $\mathrm{H}_{2} \mathrm{~S}$ [59] and $\mathrm{H}_{3} \mathrm{Se}$ [78], and slightly smaller that of $\mathrm{H}_{3} \mathrm{~S}$ [77], indicating that there is strong EPC in $\mathrm{WH}_{5}$ (Table 1). The combined projected phonon density of states (PHDOS) with Eliashberg spectral function $\alpha^{2} F(\omega)$ (Figure 3B) can be used to determine the origin of $\lambda$. For $\mathrm{WH}_{5}$, the low-frequency vibrations from the heavy $\mathrm{W}$ atoms (below $\sim 15 \mathrm{THz}$ ) contribute $38.7 \%$ of the total $\lambda$, whereas the high-frequency vibrations (above $\sim 30 \mathrm{THz}$ ) resulting from $\mathrm{H}$ atoms account for $61.3 \%$ of $\lambda$. For $\mathrm{WH}_{6}$, the low-frequency vibrations (below $\sim 15 \mathrm{THz}$ ) from the $\mathrm{W}$ atoms contribute $50 \%$ of the total $\lambda$ (Figure S3), which is in sharp contrast with $\mathrm{WH}_{5}$. The $\lambda(0.74)$ in $\mathrm{WH}_{6}$ is smaller than 0.93 in $\mathrm{WH}_{5}$, indicating a weak electron-phonon coupling in $\mathrm{WH}_{6}$. On the other hand, compared PHDOS (Figure 3B and Figure S3) between $\mathrm{WH}_{5}$ and $\mathrm{WH}_{6}$, the high-frequency and low-frequency modes of $\mathrm{WH}_{5}$ are clearly separate, unlike the situation of $\mathrm{WH}_{6}$ in which the $\mathrm{H}$-stretching vibrations mix together within the mid-frequency region. These observations can be attributed to the unique $\mathrm{H}$ network and the tetrahedron $\mathrm{H}$ units in $\mathrm{WH}_{5}$, whereas hydrogen atoms in $\mathrm{WH}_{6}$ directly bond $\mathrm{W}$ atoms (Figure $\mathrm{S4}$ ). Based above results, $\mathrm{WH}_{5}$ with unique $\mathrm{H}$ network shows good superconductivity, as will be discussed below. Similar observation has been found in $\mathrm{CaH}_{6}$ [2], $\mathrm{LaH}_{8}$ [11], and $\mathrm{YH}_{10}$ [16].

The superconducting $T_{\mathrm{c}}$ values of the stable tungsten hydrides are evaluated through the Allen-Dynes modified McMillan equation [81] by using the calculated logarithmic average

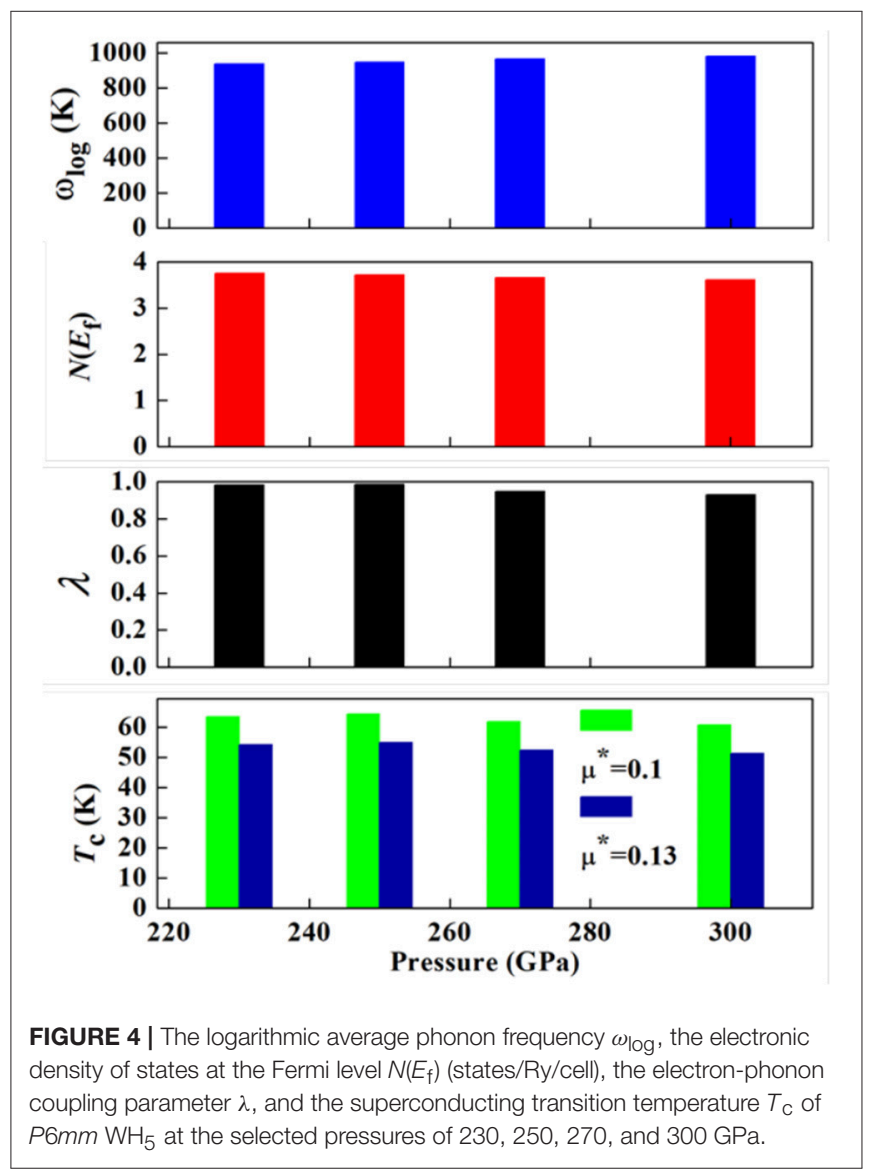

frequency, $\omega_{\log }$. The typical Coulomb pseudopotential $\mu^{*}$ usually adopts the value in the range of 0.1 to 0.13 for many metal hydrides [10]. Thus, $\mu^{*}$ with 0.1 and 0.13 was adopted here. The calculated $T_{\mathrm{c}}$ values are shown in Table 1. As expected, $\mathrm{WH}_{5}$ shows the highest $T_{c}$ value of $51.3-60.8 \mathrm{~K}$, which is attributed to its large $\lambda$ and high $\omega_{\log }$. The $T_{\mathrm{c}}$ values of $\mathrm{W}-\mathrm{H}$ compounds decrease when $\mu^{*}$ increases from 0.1 to 0.13 , similar to $\mathrm{V}-\mathrm{H}$ [36] and In-H compounds [15]. Meanwhile, we calculated the pressure-dependent $T_{\mathrm{c}}$ value of $\mathrm{WH}_{5}$. As shown in Figure 4, the effect of pressure on $T_{c}$ values is rather small. This is different from the decreased $T_{c}$ for $\mathrm{CrH}_{3}$ [82] and $\mathrm{ThH}_{10}$ [83] and the increased $T_{c}$ in $\mathrm{ScH}_{8}$ [9] with increasing pressure. In more detail, as the pressure increases, $\omega_{\log }$ becomes larger, whereas $\lambda$ becomes smaller, and $N\left(E_{\mathrm{f}}\right)$ remains nearly unchanged (Figure 4). 


\section{CONCLUSIONS}

To determine phase diagram and superconductivity of $\mathrm{W}-\mathrm{H}$ binary compounds, we have explored their crystal structures and stabilities with various chemical compositions with the aid of first-principles swarm structural calculations. We identify a hitherto unknown $\mathrm{WH}_{5}$ compound, consisting of unprecedented $\mathrm{H}$ network and tetrahedron $\mathrm{H}$ units, which can be synthesized from compression of $\mathrm{C} 2 / \mathrm{m} \mathrm{WH} 6$ and $P n m a \mathrm{WH}_{2}$ above 230.2 $\mathrm{GPa}$. All the stable tungsten hydrides are metallic in their stable pressure region. The $P 6 \mathrm{~mm} \mathrm{WH}_{5}$ shows high $T_{\mathrm{c}}$ value up to $60.8 \mathrm{~K}$ at $300 \mathrm{GPa}$, which is much higher than $\mathrm{WH}_{6}$. This work provides an opportunity for full understanding of crystal structures, electron properties, and superconducting mechanism of tungsten hydrides at megabar pressures.

\section{AUTHOR CONTRIBUTIONS}

GY and $\mathrm{AB}$ contributed conception and design of the study. ShoZ organized the database. YS, JZ, and JL performed the statistical

\section{REFERENCES}

1. Drozdov AP, Eremets MI, Troyan IA, Ksenofontov V, Shylin SI. Conventional superconductivity at 203 Kelvin at high pressures in the sulfur hydride system. Nature (2015) 525:73-6. doi: 10.1038/nature14964

2. Wang H, Tse JS, Tanaka K, Iitaka T, Ma Y. Superconductive sodalite-like clathrate calcium hydride at high pressures. Proc Natl Acad Sci USA. (2012) 109:6463-6. doi: 10.1073/pnas.1118168109

3. Li Y, Gao G, Xie Y, Ma Y, Cui T, Zou G. Superconductivity at $\sim 100 \mathrm{~K}$ in dense $\mathrm{SiH}_{4}\left(\mathrm{H}_{2}\right)_{2}$ predicted by first principles. Proc Natl Acad Sci USA. (2010) 107:15708-11. doi: 10.1073/pnas.1007354107

4. Zhong G, Zhang C, Chen X, Li Y, Zhang R, Lin H. Structural, electronic, dynamical, and superconducting properties in dense $\mathrm{GeH}_{4}\left(\mathrm{H}_{2}\right)_{2}$.J Phys Chem C (2012) 116:5225-34. doi: 10.1021/jp211051r

5. Zhou D, Jin X, Meng X, Bao G, Ma Y, Liu B, et al. Ab initio study revealing a layered structure in hydrogen-rich $\mathrm{KH}_{6}$ under high pressure. Phys Rev B Condens Matter Mater Phys. (2012) 86:14118. doi: 10.1103/PhysRevB.86.014118

6. Mao WL, Mao H -k. Hydrogen storage in molecular compounds. Proc Natl Acad Sci USA. (2004) 101:708-10. doi: 10.1073/pnas.0307449100

7. Wolverton C, Siegel DJ, Akbarzadeh AR, Ozoli V. Discovery of novel hydrogen storage materials: an atomic scale computational approach. J Phys Condens Matter (2008) 20:64228. doi: 10.1088/0953-8984/20/6/064228

8. Graetz J, Reilly JJ, Yartys VA, Maehlen JP, Bulychev BM, Antonov $\mathrm{VE}$, et al. Aluminum hydride as a hydrogen and energy storage material: past, present and future. J Alloys Comp. (2011) 509S:S517-28. doi: 10.1016/j.jallcom.2010.11.115

9. Qian S, Sheng X, Yan X, Chen Y, Song B. Theoretical study of stability and superconductivity of $\mathrm{ScH}_{\mathrm{n}}(n=4-8)$ at high pressure. Phys Rev B (2017) 96:94513. doi: 10.1103/PhysRevB.96.094513

10. Ashcroft NW. Hydrogen dominant metallic alloys: high temperature superconductors? Phys Rev Lett. (2004) 92:187002. doi: 10.1103/PhysRevLett.92.187002

11. Liu H, Naumov II, Hoffmann R, Ashcroft NW, Hemley RJ. Potential high- $\mathrm{T}_{\mathrm{c}}$ superconducting lanthanum and yttrium hydrides at high pressure. Proc Natl Acad Sci USA. (2017) 114:6990-5. doi: 10.1073/pnas.1704505114

12. Wang H, Li X, Gao G, Li Y, Ma Y. Hydrogen-Rich superconductors at high pressures. Wiley Interdiscip Rev Comput Mol Sci. (2018) 8:1-13. doi: 10.1002/wcms.1330

13. Li Y, Hao J, Liu H, Tse JS, Wang Y, Ma Y. Pressure-stabilized superconductive yttrium hydrides. Sci Rep. (2015) 5:9948. doi: 10.1038/srep09948 analysis. ShuZ wrote the first draft of the manuscript. GY and $\mathrm{AB}$ wrote sections of the manuscript. All authors contributed to manuscript revision, read and approved the submitted version.

\section{ACKNOWLEDGMENTS}

This work is supported by the Natural Science Foundation of China under Nos. 21573037, 11704062, and 51732003; the Natural Science Foundation of Jilin Province (No. 20150101042JC); the Postdoctoral Science Foundation of China (under Grant No. 2013M541283); and the Fundamental Research Funds for the Central Universities (2412017QD006).

\section{SUPPLEMENTARY MATERIAL}

The Supplementary Material for this article can be found online at: https://www.frontiersin.org/articles/10.3389/fphy. 2018.00101/full\#supplementary-material
14. Liu Y, Duan D, Huang X, Tian F, Li D, Sha X, et al. Structures and properties of osmium hydrides under pressure from first principle calculation. J Phys Chem C (2015) 119:15905-11. doi: 10.1021/acs.jpcc.5b03791

15. Liu Y, Duan D, Tian F, Liu H, Wang C, Huang X, et al. Pressure-Induced structures and properties in indium hydrides. Inorg Chem. (2015) 54:9924-8. doi: 10.1021/acs.inorgchem.5b01684

16. Peng F, Sun Y, Pickard CJ, Needs RJ, Wu Q, Ma Y. Hydrogen clathrate structures in rare earth hydrides at high pressures: possible route to room-temperature superconductivity. Phys Rev Lett. (2017) 119:107001. doi: 10.1103/PhysRevLett.119.107001

17. Geballe ZM, Liu H, Mishra AK, Ahart M, Somayazulu M, Meng Y, et al. Synthesis and stability of lanthanum superhydrides. Angew Chem Int Ed Engl. (2018) 57:688-92. doi: 10.1002/anie.201709970

18. Wigner E, Huntington HB. On the possibility of a metallic modification of hydrogen. J Chem Phys. (1935) 3:764-70. doi: 10.1063/1.1749590

19. Narayana C, Luo H, Orloff J, Ruoff AL. Solid hydrogen at 342 GPa: no evidence for an alkali metal. Nature (1998) 393:46-9. doi: 10.1038/29949

20. Dalladay-Simpson P, Howie RT, Gregoryanz E. Evidence for a new phase of dense hydrogen above 325 gigapascals. Nature (2016) 529:63. doi: 10.1038/nature16164

21. Tse JS, Yao Y, Tanaka K. Novel superconductivity in metallic $\mathrm{SnH}_{4}$ under high pressure. Phys Rev Lett. (2007) 98:117004. doi: 10.1103/PhysRevLett.98.117004

22. Ye X, Zarifi N, Zurek E, Hoffmann R, Ashcroft NW. High hydrides of scandium under pressure: potential superconductors. J Phys Chem C (2018) 122:6298-309. doi: 10.1021/acs.jpcc.7b12124

23. Ashcroft NW. Metallic hydrogen: a high-temperature superconductor? Phys Rev Lett. (1968) 21:1748. doi: 10.1103/PhysRevLett.21.1748

24. Bi T, Zarifi N, Terpstra T, Zurek E. The search for superconductivity in high pressure hydrides. arXiv [Preprint] arXiv (2018) 1806:163.

25. Gao G, Hoffmann R, Ashcroft NW, Liu H, Bergara A, Ma Y. Theoretical study of the ground-state structures and properties of niobium hydrides under pressure. Phys Rev B (2013) 88:184104. doi: 10.1103/PhysRevB.88.184104

26. Liu Y, Huang X, Duan D, Tian F, Liu H, Li D, et al. First-principles study on the structural and electronic properties of metallic $\mathrm{HfH}_{2}$ under pressure. Sci Rep. (2015) 5:11381. doi: 10.1038/srep11381

27. Shanavas KV, Lindsay L, Parker DS. Electronic structure and electron-phonon coupling in $\mathrm{TiH}_{2}$. Sci Rep. (2016) 6:28102. doi: 10.1038/srep28102

28. Wang L, Duan D, Yu H, Xie H, Huang X, Ma Y, et al. High-Pressure formation of cobalt polyhydrides: a first-principle study. Inorg Chem. (2018) 57:181-6. doi: 10.1021/acs.inorgchem.7b02371 
29. Feng X, Zhang J, Gao G, Liu H, Wang H. Compressed sodalite-like $\mathrm{MgH}_{6}$ as a potential high-temperature superconductor. RSC Adv. (2015) 5:59292. doi: 10.1039/C5RA11459D

30. Zhang $\mathrm{H}$, Jin $\mathrm{X}$, Lv $\mathrm{Y}$, Zhuang Q, Liu Y, Lv Q, et al. A novel stable hydrogen-rich $\mathrm{SnH}_{8}$ under high pressure. RSC Adv. (2015) 5:107637. doi: 10.1039/c5ra20428c

31. Xie Y, Li Q, Oganov AR, Wang H. Superconductivity of lithiumdoped hydrogen under high pressure. Acta Crystallogr Sect C Struct Chem. (2014) 70:104-11. doi: 10.1107/S20532296130 28337

32. Wiedner ES, Chambers MB, Pitman CL, Bullock RM, Miller AJM, Appel AM. Thermodynamic hydricity of transition metal hydrides. Chem Rev. (2016) 116:8655-92. doi: 10.1021/acs.chemrev.6b00168

33. Abe K. Hydrogen-Rich scandium compounds at high pressures. Phys Rev B (2017) 96:144108. doi: 10.1103/PhysRevB.96.1 44108

34. Scheler T, Marqués M, Konôpková Z, Guillaume CL, Howie RT, Gregoryanz E. High-Pressure synthesis and characterization of iridium trihydride. Phys Rev Lett. (2013) 111:215503. doi: 10.1103/PhysRevLett.111.2 15503

35. Zhuang Q, Jin X, Cui T, Ma Y, Lv Q, Li Y, et al. Pressure-Stabilized superconductive ionic tantalum hydrides. Inorg Chem. (2017) 56:3901-8. doi: 10.1021/acs.inorgchem.6b02822

36. Li X, Peng F. Superconductivity of pressure-stabilized vanadium hydrides. Inorg Chem. (2017) 56:13759-65. doi: 10.1021/acs.inorgchem.7b01686

37. Pépin CM, Dewaele A, Geneste G, Loubeyre P, Mezouar M. New iron hydrides under high pressure. Phys Rev Lett. (2014) 113:265504. doi: 10.1103/PhysRevLett.113.265504

38. Sumuraya TT, Atsuura YM, Hishidou TS, Guchi TO. First-Principles study on the structural and magnetic properties of iron hydride. J Phys Soc Jpn. (2012) 81:064707. doi: 10.1143/JPSJ.81.064707

39. Bazhanova ZG, Oganov AR, Gianola O. Fe-C and $\mathrm{Fe}-\mathrm{H}$ systems at pressures of the earth's inner core. Phys Uspekhi (2012) 55:489-97. doi: 10.3367/UFNe.0182.201205c.0521

40. Li F, Wang D, Du H, Zhou D, Ma Y, Liu Y. Structural evolution of $\mathrm{FeH}_{4}$ under high pressure. RSC Adv. (2017) 7:12570-5. doi: 10.1039/C6RA25591D

41. Pépin CM, Geneste G, Dewaele A, Mezouar M, Loubeyre P. Synthesis of FeH : a layered structure with atomic hydrogen slabs. Science (2017) 357:382-5. doi: 10.1126/science.aan0961

42. Majumdar A, Tse JS, Wu M, Yao Y. Superconductivity in $\mathrm{FeH}_{5}$. Phys Rev B (2017) 96:201107. doi: 10.1103/PhysRevB.96.201107

43. Kvashnin AG, Kruglov IA, Semenok DV, Oganov AR. Iron superhydrides $\mathrm{FeH}_{5}$ and $\mathrm{FeH}_{6}$ : stability, electronic properties, and superconductivity. J Phys Chem C (2018) 122:4731-6. doi: 10.1021/acs.jpcc.8b01270

44. Zhang S, Lin J, Wang Y, Yang G, Bergara A, Ma Y. Nonmetallic $\mathrm{FeH}_{6}$ under high pressure. J Phys Chem C (2018) 122:12022-8. doi: 10.1021/acs.jpcc.7b11643

45. Kim DY, Scheicher RH, Ahuja R. Predicted high-temperature superconducting state in the hydrogen-dense transition-metal hydride $\mathrm{YH}_{3}$ at $40 \mathrm{~K}$ and 17.7GPa. Phys Rev Lett. (2009) 103:77002. doi: 10.1103/PhysRevLett.103.077002

46. Wang X, Andrews L. Neon matrix infrared spectra and DFT calculations of tungsten hydrides $\mathrm{WH}_{\mathrm{x}}(\mathrm{x}=1-4,6)$. J Phys Chem A (2002) 106:6720-9. doi: $10.1021 /$ jp025920d

47. Wang X, Andrews L, Infante I, Gagliardi L. Infrared spectra of the $\mathrm{WH}_{4}\left(\mathrm{H}_{2}\right)_{4}$ complex in solid hydrogen. J Am Chem Soc. (2008) 130:1972-8. doi: $10.1021 /$ ja077322o

48. Arnoult, William J, McLellan RB. Thermodynamics of transition metal alloys. Acta Metall. (1973) 21:1397.

49. Strobel TA, Somayazulu M, Hemley RJ. Novel pressure-induced interactions in silane-hydrogen. Phys Rev Lett. (2009) 103:65701. doi: 10.1103/PhysRevLett.103.065701

50. Zaleski-Ejgierd P, Labet V, Strobel TA, Hoffmann R, Ashcroft NW. $\mathrm{WH}_{\mathrm{n}}$ under pressure. J Phys Condens Matter (2012) 24:155701. doi: 10.1088/0953-8984/24/15/155701

51. Wang $\mathrm{X}$, Andrews L. Neon matrix infrared spectrum of $\mathrm{WH}_{6}$ : a distorted trigonal prism structure. J Am Chem Soc. (2002) 124:5636-7. doi: $10.1021 /$ ja020216w
52. Scheler T, Peng F, Guillaume CL, Howie RT, Ma Y, Gregoryanz E. Nanocrystalline tungsten hydrides at high pressures. Phys Rev B (2013) 87:184117. doi: 10.1103/PhysRevB.87.184117

53. Hooper J, Altintas B, Shamp A, Zurek E. Polyhydrides of the alkaline earth metals: a look at the extremes under pressure. J Phys Chem C (2013) 117:298292. doi: 10.1021/jp311571n

54. Zhong X, Wang H, Zhang J, Liu H, Zhang S, Song HF, et al. Tellurium hydrides at high pressures: high-temperature superconductors. Phys Rev Lett. (2016) 116:57002. doi: 10.1103/PhysRevLett.116.057002

55. Wang Y, Lv J, Zhu L, Ma Y. Crystal structure prediction via particle-swarm optimization. Phys Rev B Condens Matter Mater Phys. (2010) 82:94116. doi: 10.1103/PhysRevB.82.094116

56. Wang Y, Lv J, Zhu L, Ma Y. CALYPSO: a method for crystal structure prediction. Comput Phys Commun. (2012) 183:2063-70. doi: 10.1016/j.cpc.2012.05.008

57. Zhu L, Wang H, Wang Y, Lv J, Ma Y, Cui Q, et al. Substitutional alloy of $\mathrm{Bi}$ and Te at high pressure. Phys Rev Lett. (2011) 106:145501. doi: 10.1103/PhysRevLett.106.145501

58. Lv J, Wang Y, Zhu L, Ma Y. Predicted novel high-pressure phases of lithium. Phys Rev Lett. (2011) 106:15503. doi: 10.1103/PhysRevLett.106.015503

59. Li Y, Hao J, Liu H, Li Y, Ma Y. The metallization and superconductivity of dense hydrogen sulfide. J Chem Phys. (2014) 140:174712. doi: $10.1063 / 1.4874158$

60. Zhu L, Liu H, Pickard CJ, Zou G, Ma Y. Reactions of xenon with iron and nickel are predicted in the earth's inner core. Nat Chem. (2014) 6:644-8. doi: 10.1038/nchem.1925

61. Yang G, Wang Y, Peng F, Bergara A, Ma Y. Gold as a 6p-element in dense lithium aurides. J Am Chem Soc. (2016) 138:4046-52. doi: 10.1021 /jacs.5b11768

62. Zhang S, Zhu L, Liu H, Yang G. Structure and electronic properties of $\mathrm{Fe}_{2} \mathrm{SH}_{3}$ compound under high pressure. Inorg Chem. (2016) 55:11434-9. doi: 10.1021/acs.inorgchem.6b01949

63. Pfrommer BG, Côté M, Louie SG, Cohen ML. Relaxation of crystals with the quasi-newton method. J Comput Phys. (1997) 131:233-40. doi: $10.1006 /$ jcph.1996.5612

64. Clark SJ, Segall MD, Pickard CJ, Hasnip PJ, Probert MJ, Refson K, et al. First principles methods using CASTEP. Zeitschrift fuer Krist (2005) 220:567-70. doi: 10.1524/zkri.220.5.567.65075

65. Perdew J, Chevary J, Vosko S, Jackson K, Pederson M, Singh D, et al. atoms, molecules, solids, and surfaces: applications of the generalized gradient approximation for exchange and correlation. Phys Rev B Condens Matter Mater Phys. (1992) 46:6671. doi: 10.1103/PhysRevB.48.4978.2

66. Vanderbilt D. Soft self-consistent pseudopotentials in a generalized eigenvalue formalism. Phys Rev B (1990) 41:7892-5. doi: 10.1103/PhysRevB.41.7892

67. Pack JD, Monkhorst HJ. Special points for brillouin-zone integrations. Phys Rev B (1976) 13:5188. doi: 10.1103/PhysRevB.16.1748

68. Giannozzi P, Baroni S, Bonini N, Calandra M, Car R, Cavazzoni C, et al QUANTUM ESPRESSO: a modular and open-source software project for quantum simulations of materials. J Phys Condens Matter (2009) 21:395502. doi: 10.1088/0953-8984/21/39/395502

69. Deshpande VT, Pawar R. X-Ray Determination of the thermal expansion of tungsten. Curr Sci. (1962) 31:497-9.

70. Pickard CJ, Needs RJ. Structure of phase III of solid hydrogen. Nat Phys. (2007) 3:473-6. doi: 10.1038/nphys625

71. Zhuang Q, Jin X, Lv Q, Li Y, Shao Z, Liu Z, et al. Investigation of superconductivity in compressed vanadium hydrides. Phys Chem Chem Phys. (2017) 19:26280. doi: 10.1039/C7CP03435K

72. Segall M, Shah R, Pickard C, Payne M. Population analysis of planewave electronic structure calculations of bulk materials. Phys Rev B (1996) 54:16317-20. doi: 10.1103/PhysRevB.54.16317

73. Marck SC. Site percolation and random walks on d-Dimensional kagomé lattices. J Phys Math Gen. (1998) 31:3349-460. doi: 10.1088/0305-4470/31/15/010

74. Atwood JL. Kagomé lattice: a molecular toolkit for magnetism. Nat Mater. (2002) 1:91. doi: 10.1038/nmat740

75. Schlickum U, Decker R, Klappenberger F, Zoppellaro G, Klyatskaya S, Auwärter W, et al. Chiral kagomé lattice from simple ditopic molecular bricks. J Am Chem Soc. (2008) 130:11778-82. doi: 10.1021/ja8028119 
76. McMahon JM, Ceperley DM. Ground-State structures of atomic metallic hydrogen. Phys Rev Lett. (2011) 106:165302. doi: 10.1103/PhysRevLett.106.1 65302

77. Duan D, Liu Y, Tian F, Li D, Huang $\mathrm{X}$, Zhao Z, et al. Pressure-Induced metallization of dense $\left(\mathrm{H}_{2} \mathrm{~S}\right)_{2} \mathrm{H}_{2}$ with high$\mathrm{T}_{c}$ superconductivity. Sci Rep. (2015) 4:6968. doi: 10.1038/srep0 6968

78. Zhang S, Wang Y, Zhang J, Liu H, Zhong X, Song HF, et al. Phase diagram and high-temperature superconductivity of compressed selenium hydrides. Sci Rep. (2015) 5:15433. doi: 10.1038/srep 15433

79. Arndt Simon. Superconductivity and Chemistry. Angew Chem Int Ed Engl. (1997) 36:1788-1806.

80. Bardeen J, Cooper LN, Schrieffer JR. Theory of superconductivity. Phys Rev. (1957) 108:1175. doi: 10.1103/PhysRev.108.1175

81. Allen PB, Dynes RC. Transition temperature of strong-couple superconductors reanalyzed. Phys Rev B (1975) 12:905-22. doi: 10.1103/PhysRevB.12.905
82. Yu S, Jia X, Frapper G, Li D, Oganov AR, Zeng Q, et al. Pressure-Driven formation and stabilization of superconductive chromium hydrides. Sci Rep. (2015) 5:17764. doi: 10.1038/srep17764

83. Kvashnin AG, Semenok DV, Kruglov IA, Oganov AR. High-Temperature superconductivity in Th-H system at pressure conditions. arXiv [Preprint] $\operatorname{arXiv}(2017)$ 1711:00278.

Conflict of Interest Statement: The authors declare that the research was conducted in the absence of any commercial or financial relationships that could be construed as a potential conflict of interest.

Copyright (c) 2018 Zheng, Zhang, Sun, Zhang, Lin, Yang and Bergara. This is an open-access article distributed under the terms of the Creative Commons Attribution License (CC BY). The use, distribution or reproduction in other forums is permitted, provided the original author(s) and the copyright owner(s) are credited and that the original publication in this journal is cited, in accordance with accepted academic practice. No use, distribution or reproduction is permitted which does not comply with these terms. 